pag

Business School

WORKING PAPER SERIES

Working Paper

2014-329
Dynamic Portfolio Insurance Strategies: Risk Management under Johnson Distributions

Naceur Naguez

Jean-Luc Prigent

http://www.ipag.fr/fr/accueil/la-recherche/publications-WP.html

IPAG Business School

184, Boulevard Saint-Germain

75006 Paris

France 


\title{
Dynamic Portfolio Insurance Strategies: Risk Management under Johnson Distributions
}

\author{
Naceur Naguez* Jean-Luc Prigent ${ }^{\dagger}$
}

This version: May 2014.

\begin{abstract}
The purpose of this paper is to analyze the gap risk of dynamic portfolio insurance strategies which generalize the "Constant Proportion Portfolio Insurance " (CPPI) method by allowing the multiple to vary. We illustrate our theoretical results for conditional CPPI strategies indexed on hedge funds. For this purpose, we provide accurate estimations of hedge funds returns by means of Johnson distributions. We introduce also an EGARCH type model with Johnson innovations to describe dynamics of risky logreturns. We use both VaR and Expected Shortfall as downside risk measures to control gap risk. We provide accurate upper bounds on the multiple in order to limit this gap risk. We illustrate our theoretical results on Credit Suisse Hedge Fund Index. The time period of the analysis lies between December 1994 and December 2013.
\end{abstract}

Keywords: Portfolio insurance; CPPI; Hedge funds; Johnson distribution, gap risk, VaR, CVaR.

JEL classification: C6, G11, G24, L10.

*University of Cergy-Pontoise, THEMA. 33 Bd du Port, 95011, Cergy-Pontoise, France. E-mail: naceur.naguez@u-cergy.fr

${ }^{\dagger}$ IPAG Business School and University of Cergy-Pontoise, THEMA. 33 Bd du Port, 95011, Cergy-Pontoise, France. E-mail: jean-luc.prigent@u-cergy.fr 


\section{Introduction}

Alternative investments have enjoyed increasing success with institutional and private investors around the world. This phenomenon of globalization has been fostered by poor market conditions of the 2000s which have illustrated the advantages of this style of portfolio management compared to traditional management. Providing returns that are decorrelated with standard market indices, hedge funds have become attractive for many fund managers. Hedge fund performances can potentially provide benefits to individuals who, either invest directly in these funds, either invest in portfolios of hedge funds managed by portfolio insurance methods (as recently illustrated).

The goal of portfolio insurance is first to limit downside risk in bearish financial markets, second to allow some participation in bullish markets. There exist several methods of portfolio insurance: OBPI (Option Based Portfolio Insurance), CPPI (Constant Proportion Portfolio Insurance), Stop-loss, ... (see for example Poncet and Portait (1997) and Bertrand and Prigent (2011)for a review about these methods). Here, we examine the CPPI method introduced by Black and Jones (1987) for equity instruments and Perold (1986), Black and Perold (1988) for fixed-income instruments (see also Black and Rouhani (1987), Roman, Kopprash and Hakanoglu (1989), Black and Perold (1992)). The CPPI method uses a simplified strategy to allocate assets dynamically over time. Basically, two assets are involved: the riskless asset, $B$, with a constant interest rate $r$ (usually Treasury bills or other liquid money market instruments) and the risky one, $S$.

Usually, the investor, with initial amount to invest $V_{0}$, wants to recover a fixed percentage $p$ of his initial investment at a given horizon $T$. To obtain a terminal portfolio value $V_{T}$ higher than the insured amount $p V_{0}$, the portfolio manager keeps the portfolio value $V_{t}$ above the floor $F_{t}=p \cdot V_{0} \cdot e^{-r(T-t)}$ at any time $t$ in the period $[0, T]$. For this purpose, the amount $e_{t}$ (called the exposure) invested in the risky asset is equal to a fixed proportion $m$ (called the multiple) of the excess of the portfolio value over the floor, also called the cushion and thus defined by $C_{t}=V_{t}-F_{t}$. Therefore, this insurance method consists in keeping $C_{t}$ positive at any time $t$ in the period. The remaining funds are invested in the riskless asset $B_{t}$.

Advantages of this portfolio insurance strategy over other approaches are its simplicity and its flexibility. Initial cushion, floor and tolerance can be determined according to the own investor's objective (see Poncet and Portait, 1997). Issuers (banks or insurance companies) may bear market risks on the insured portfolios, due to sudden large market drops. For instance, in the case of the CPPI method, issuers must at least provision the difference with their own capital if the value of the portfolio becomes smaller than the floor. Therefore, they can use, for example, stress testing to estimate their potential losses.

Thus, the issuer faces one crucial question: what exposure to the risky asset or, equivalently, what level of the multiple to accept? On one hand, since 
portfolio return expectation is increasing with respect to the multiple, customers want to benefit from a multiple as high as possible. On the other hand, due to market imperfections, ${ }^{1}$ portfolio managers must impose an upper bound on the multiple. For example, if the portfolio manager anticipates that the maximal daily historical drop (e.g. -20\%) will occur during the period, he chooses $m \leq 5$ which leads to low return expectation. Alternatively, he may consider that the maximal daily drop during the management period will never be higher than a given value (e.g. $-10 \%)$. A straightforward implication is to choose $m$ according to this new extreme value (e.g. $m \leq 10$ ). Another possibility is to take account of the occurrence probabilities of extreme events in the risky asset returns. Then, the portfolio manager can adopt a quantile hedging strategy by determinating the maximum multiple so that the portfolio value will always be above the floor at a given probability level (typically 99\%).

This latter management approach has important practical implications. The determination of the multiple according to quantile conditions have been examined for instance in Prigent (2001) in the case of a Lévy process and in Bertrand and Prigent (2002), using extreme value theory. Hamidi et al (2009a,b) and Ben Ameur and Prigent (2014) also consider a modified quantile hedging strategy and show how a conditional multiple can be determined. For this purpose, Hamidi et al (2014) introduce quantile regressions, like for example Engel and Manganelli (2004) who study Conditional Auto Regressive Value-at-Risk by Regression Quantile (CAViaR).

In this paper, we show how the portfolio manager can choose the value of the multiple according to hedge funds return fluctuations. This allows the introduction of a conditional multiple. To illustrate our gap risk management approach, we consider both quantile and expected shortfall conditions. We consider a quite general Johnson distribution to model hedge funds returns. Our results prove that, in the generalized Johnson framework, a conditional multiple can be determined as functions of state variables such as the past returns and volatilities.

The paper is organized as follows. Section 2 introduces the modelling of hedge funds returns by Johnson distributions. Section 3 presents the basic properties of the CPPI model in this framework. Section 4 details the modified CPPI method based on a conditional multiple that is determined from quantile and expected shortfall conditions. In particular, upper bounds on the multiple are provided. Section 5 provides the empirical analysis. Some proofs are gathered in Appendix.

\footnotetext{
${ }^{1}$ For example, it is well known that portfolio managers cannot actually modify their portfolio weights in continuous time. Additionally, asset liquidity problems may occur, especially during stock markets crashes (see Longin, 1997).
} 


\section{Modelling hedge funds returns}

Many studies about hedge fund returns have shown that the mean-variance approach is not adequate to evaluate the risks and performances of these specific funds. Indeed, hedge funds returns distributions can have negative skewness and excess kurtosis, as documented by Fung and Hsieh (1997), Ackerman et al. (1999), Brown et al. (1999), Brooks and Kat (2001), Caglayan and Edwards (2001), Bacmann and Scholz (2003), Agarwal and Naik (2004)...This leads to conclude that, for this type of fund, the challenge lies not only in the first two moments but also in higher moments. In this framework, the selection of a family of distributions with sufficient flexibility to take account of the asymmetry and of the kurtosis is necessary to study the behavior of hedge funds returns. The Johnson distribution system (1949) satisfies this requirement.

\subsection{Families of Johnson distributions}

\subsubsection{Johnson system}

The Johnson system covers three main families of probability distributions that can model a wide variety of empirical distributions. Additionally, we can introduce a quite general family of probability distributions that we call the Generalized Johnson Distributions (GJD) defined as follows. Given a continuous random variable $X, X$ has a Johnson distribution if there exists a monotonic and invertible function $g$ such that:

$$
\varepsilon=\gamma+\delta g\left(\frac{X-\xi}{\lambda}\right),
$$

where $\varepsilon$ is a standard normal random variable, and $\gamma, \delta, \xi, \lambda$ are four given parameters. Usually, $\gamma$ and $\delta$ correspond to trend parameters, $\xi$ is a positional parameter and finally $\lambda$ corresponds to a scaling parameter.

Notation 1 We introduce the function $h$ and its inverse by setting:

$$
\begin{aligned}
h(x) & =\gamma+\delta g\left(\frac{x-\xi}{\lambda}\right), \\
h^{-1}(z) & =\lambda g^{-1}\left(\frac{z-\gamma}{\delta}\right)+\xi .
\end{aligned}
$$

and also $y=\left(\frac{x-\xi}{\lambda}\right)$.

The three standard types of Johnson distribution correspond to a function $g($.$) defined by:$

- A lognormal distribution

$$
S_{L}: g(y)=\ln (y) .
$$

- An unbounded distribution 


$$
S_{U}: g(y)=\ln \left(y+\sqrt{1+y^{2}}\right)=\sinh ^{-1}(y) .
$$

- A bounded distribution

$$
S_{B}: g(y)=\ln \left(\frac{y}{1-y}\right) .
$$

The $S_{U}$ distribution is unbounded in both directions while the $S_{B}$ distribution is bounded in both directions $(\xi, \xi+\lambda)$. The Johnson probability density function (pdf) $f_{X}$ is easily deduced from standard normal distribution. It is given by canonical transformations of the standard Gauss distribution:

$$
\begin{gathered}
f_{X}(x)= \\
\frac{\delta}{\lambda \sqrt{2 \pi}} \exp \left[-\frac{1}{2}\left\{\gamma+\delta g\left(\frac{x-\xi}{\lambda}\right)\right\}^{2}\right]\left|g^{\prime}\left(\frac{x-\xi}{\lambda}\right)\right| .
\end{gathered}
$$

The pdf $f_{\widetilde{X}}$ corresponding to each of the three main Johnson distributions is also easily deduced from previous general form:

$$
\begin{gathered}
S_{L}: f_{X}(x)= \\
\frac{\delta}{\lambda \sqrt{2 \pi}\left(\frac{x-\xi}{\lambda}\right)} \exp \left[-\frac{1}{2}\left\{\gamma+\delta \ln \left(\frac{x-\xi}{\lambda}\right)\right\}^{2}\right] \text { for } \frac{x-\xi}{\lambda}<0 . \\
\frac{\delta}{\lambda \sqrt{2 \pi} \sqrt{\left(\frac{x-\xi}{\lambda}\right)^{2}+1}} \exp \left[-\frac{1}{2}\left\{\gamma+\delta \ln \left(\frac{x-\xi}{\lambda}+\sqrt{\left(\frac{x-\xi}{\lambda}\right)^{2}+1}\right)\right\}^{2}\right] . \\
\quad S_{B}: f_{X}(x)= \\
\text { for } 0<\frac{\delta}{\lambda \sqrt{2 \pi}\left(\frac{x-\xi}{\lambda}\right)\left(1-\frac{x-\xi}{\lambda}\right)} \exp \left[\frac{1}{2}\left\{\gamma+\delta \ln \left(\frac{\frac{x-\xi}{\lambda}}{1-\left(\frac{x-\xi}{\lambda}\right)}\right)\right\}^{2}\right] \\
\text {. }
\end{gathered}
$$

When using Johnson type distributions, we can:

- First search among the three families of Johnson distributions to be introduced;

- Second estimate the parameters of this distribution;

- Finally, if necessary, determine the best function $g$, using a non parametric approach. In that latter case, function $g$ may no longer belong to one of the three basic families. 


\section{CPPI with fixed multiple and Johnson distri- butions}

We begin by briefly recalling the main properties of the standard CPPI strategy in discrete time (constant multiple).

\subsection{The financial market}

Consider two basic financial assets: a riskless asset, denoted by $B$, and a hedge fund denoted by $S$. Changes in asset prices are supposed to occur at discrete times along a whole management period $[0, T]$. This assumption is in accordance to actual practice when investing on hedge funds.

The riskless asset evolves according to a deterministic rate denoted by $r$.

The variations of the hedge funds $S$ between two times $t_{k-1}$ and $t_{k}$ are defined by:

$$
\Delta S_{t_{k+1}}=S_{t_{k+1}}-S_{t_{k}} .
$$

Since we search for an upper bound on the multiple $m$, we have to focus on the left hand side of the probability distribution of $\frac{\Delta S_{t_{k}}}{S_{t_{k-1}}}$.

Denote also:

$$
R_{t_{k}}=\left(\frac{S_{t_{k}}}{S_{t_{k-1}}}\right) \text { and } Z_{t_{k}}=\ln \left(R_{t_{k}}\right)
$$

\subsection{The CPPI portfolio}

Denote by $V_{t_{k}}$ the value of the portfolio at time $t_{k}$. As explained in introduction, the CPPI method is based on the following portfolio insurance conditions:

- There exists a deterministic floor $F_{t_{k}}$ such that at any time $t_{k}$, the value $V_{t_{k}}$ must be above the floor.

- The total amount $e_{t_{k}}$ invested on the underlying asset $S$ is equal to $m C_{t_{k}}$ where the cushion $C_{t_{k}}$ represents the difference $V_{t_{k}}-F_{t_{k}}$, between the portfolio value and the floor.

- The multiple $m$ is a non negative constant.

- The remaining amount $\left(V_{t_{k}}-e_{t_{k}}\right)$ is invested on the riskless asset with a deterministic rate $r_{t_{k}}$ for the period $\left[t_{k-1}, t_{k}\right]$.

The higher the multiple $m$, the higher the amount invested on the risky asset. Therefore, a speculative investor would choose high values for $m$. Nevertheless, in that case, his portfolio is riskier and as shown in what follows, his guarantee may no longer hold. Indeed, we easily deduce that the portfolio value is solution of

$$
V_{t_{k+1}}=V_{t_{k}}+e_{t_{k}}\left(\frac{\Delta S_{t_{k+1}}}{S_{t_{k}}}\right)+\left(V_{t_{k}}-e_{t_{k}}\right) r_{t_{k+1}}
$$


from which, we deduce the dynamics of the cushion:

$$
C_{t_{k+1}}=C_{t_{k}}\left[1+m\left(\frac{\Delta S_{t_{k+1}}}{S_{t_{k}}}\right)+(1-m) r_{t_{k+1}}\right] .
$$

Since, for all times $t_{k}$, the cushion must be positive, we get finally the condition: for all $k \leq n$,

$$
m\left(\frac{\Delta S_{t_{k+1}}}{S_{t_{k}}}\right)+(1-m) r_{t_{k}} \geq-1
$$

In fact, since $r_{t_{k}}$ is relatively small, the previous inequality yields to the following relation that gives an upper bound on the multiple:

Proposition 2 The guarantee is satisfied at any time of the management period with a probability equal to 1 if and only if

$$
\forall k \leq n,-\frac{\Delta S_{t_{k+1}}}{S_{t_{k}}} \leq \frac{1}{m} \text { or equivalently } M_{n}=\operatorname{Max}\left(-\frac{\Delta S_{t_{k+1}}}{S_{t_{k}}}\right)_{k \leq n} \leq \frac{1}{m},
$$

which is equivalent to:

$$
\operatorname{Max}\left(1-R_{t_{k}}\right) \leq \frac{1}{m}, \text { and also } \operatorname{Min}\left(R_{t_{k}}\right) \geq\left(1-\frac{1}{m}\right) .
$$

Let us now deeply examine this condition when the return on the risky asset follows a generalized Johnson distribution.

Denote by $\epsilon_{t_{k}}$ the logarithm of the normalized return conditionally on the available information at time $t_{k-1}$ :

$$
\epsilon_{t_{k}}=\frac{\ln R_{t_{k}}-\mu_{t_{k}}}{\sigma_{t_{k}}}
$$

Thus, at any time $t_{k+1}$ the risky asset value is given by:

$$
S_{t_{k+1}}=S_{t_{k}} \exp \left[\mu_{t_{k}}+\sigma_{t_{k}} \epsilon_{t_{k}}\right] .
$$

Assuming that the probability distribution of the innovation process $\epsilon_{t_{k}}$ is a generalized Johnson distribution, we have:

$$
\varepsilon_{t_{k}}=h_{t_{k}}\left(\epsilon_{t_{k}}\right) \text { where } h_{t_{k}}(x)=\gamma_{t_{k}}+\delta_{t_{k}} g\left(\frac{x-\xi_{t_{k}}}{\lambda_{t_{k}}}\right),
$$

where the four parameters $\gamma_{t_{k}}, \delta_{t_{k}}, \xi_{t_{k}}, \lambda_{t_{k}}$ are deterministic. We get equivalently:

$$
\epsilon_{t_{k}}=h_{t_{k}}^{-1}\left(\varepsilon_{t_{k}}\right) \text { where } h_{t_{k}}^{-1}(z)=\lambda_{t_{k}} g^{-1}\left(\frac{z-\gamma_{t_{k}}}{\delta_{t_{k}}}\right)+\xi_{t_{k}} \text {. }
$$

Then we deduce a general insurance condition. 
Proposition 3 The guarantee is satisfied at any time $t_{k}$ of the management period with a probability equal to 1 if and only if

$$
\operatorname{Min}\left(\varepsilon_{t_{k}}\right) \geq \delta_{t_{k}} g\left(\frac{\left[\frac{\ln \left(1-\frac{1}{m}\right)-\mu_{t_{k}}}{\sigma_{t_{k}}}\right]-\xi_{t_{k}}}{\lambda_{t_{k}}}\right)+\gamma_{t_{k}} .
$$

Remark 4 Since the right end point $d$ of the common distribution of the variables $\left(-\frac{\Delta S_{t_{k+1}}}{S_{t_{k}}}\right)$ is positive, we deduce that the insurance is perfect along any period $[0, T]$ if and only if $m$ is smaller than $\frac{1}{d}$. For example, if the maximal drop is equal to $20 \%$, then $d=0,2$. Thus $m$ must be smaller than 5 .

\subsection{Quantile condition with a fixed multiple}

Previous condition (8) is rather stringent. Therefore, we can search for an alternative rationale condition like for example a criterion such as the Valueat-Risk. This latter approach is based on quantile hedging as emphasized by Föllmer and Leukert (1999).

In that case, the new guarantee condition is that the portfolio value is always higher than the floor at a given probability $1-a$ (for example $a=1 \%$ ). This yields to the following relation for a management time period $[0, T]$ :

$$
\mathbb{P}\left[C_{t_{k}} \geq 0, \forall t_{k} \in\{1, \ldots, T\}\right] \geq 1-a,
$$

which is equivalent to:

$$
\mathbb{P}\left[\forall t_{k} \in\{1, \ldots, T\},-\frac{\Delta S_{t_{k+1}}}{S_{t_{k}}} \leq \frac{1}{m}\right] \geq 1-a .
$$

In the generalized Johnson distributions framework, this leads to the following condition:

$$
\mathbb{P}\left[\forall t_{k} \in\{1, \ldots, T\}, \varepsilon_{t_{k}} \geq \delta_{t_{k}} g\left(\frac{\left[\frac{\ln \left(1-\frac{1}{m}\right)-\mu_{t_{k}}}{\sigma_{t_{k}}}\right]-\xi_{t_{k}}}{\lambda_{t_{k}}}\right)+\gamma_{t_{k}}\right] \geq 1-a .
$$

Denote by $N$ the cdf of the standard Gaussian distribution. If $\left(\varepsilon_{t_{k}}\right)_{k}$ is i.i.d. (recall that $g$ is increasing), then Condition (10) is equivalent to:

$$
1-N\left[\delta_{t_{k}} g\left(\frac{\left[\frac{\ln \left(1-\frac{1}{m}\right)-\mu_{t_{k}}}{\sigma_{t_{k}}}\right]-\xi_{t_{k}}}{\lambda_{t_{k}}}\right)+\gamma_{t_{k}}\right] \geq(1-a)^{\frac{1}{T}},
$$


from which we deduce:

$$
N\left[\delta_{t_{k}} g\left(\frac{\left[\frac{\ln \left(1-\frac{1}{m}\right)-\mu_{t_{k}}}{\sigma_{t_{k}}}\right]-\xi_{t_{k}}}{\lambda_{t_{k}}}\right)+\gamma_{t_{k}}\right] \leq 1-(1-a)^{\frac{1}{T}} .
$$

Therefore, for the i.i.d. case, we can characterize the quantile condition.

Proposition 5 For small $r_{t_{k}}$ values and if the sequence $\left(\varepsilon_{t_{k}}\right)_{k}$ is i.i.d. with common cdf $N$, then we have (approximately) the following condition:

$$
\delta_{t_{k}} g\left(\frac{\left[\frac{\ln \left(1-\frac{1}{m}\right)-\mu_{t_{k}}}{\sigma_{t_{k}}}\right]-\xi_{t_{k}}}{\lambda_{t_{k}}}\right)+\gamma_{t_{k}} \leq q_{a, T} \text {, with } q_{a, T}=N^{-1}\left[1-(1-a)^{\frac{1}{T}}\right] \text {, }
$$

which provides the following upper bound on the multiple:

$$
m \leq \operatorname{Min}_{k}\left[\frac{1}{1-\exp \left[h_{t_{k}}^{-1}\left(q_{a, T}\right) \sigma_{t_{k}}+\mu_{t_{k}}\right]}\right] .
$$

Remark 6 Note that this upper limit on the multiple $m$ is obviously higher than the standard limit $\frac{1}{d}$.

For a given probability level a anf for a given transformation $h_{t_{k}}$, this upper bound depends on the two state variables, namely the conditional trend $\mu_{t_{k}}$ and the conditional volatility $\sigma_{t_{k}}$.

It is an increasing function with respect to the conditional trend, while, for standard values of the probability level $\alpha\left(q_{a, T}<0\right)$, it is a decreasing function of the conditional volatility.

As intuitively, the higher the conditional return expectation, the less stringent the quantile condition ((i.e. the higher the upper bound); the higher the conditional volatility, the more stringent the quantile condition (i.e. the smaller the upper bound). 


\section{CPPI with a conditional multiple and John- son distributions}

In previous section, the multiple is assumed to be constant. It is chosen and fixed at initial time of the management period. In what follows, we allow the multiple to vary according to market fluctuations, in order to get sufficient flexibility to improve portfolio performance.

\subsection{Conditional multiple}

The CPPI method is sufficiently flexible so that several extensions can be proposed.

- For example, Estep and Kritzman (1988) have introduced the Time Invariant Portfolio Protection (TIPP), which is based on a stochastic floor to keep past profits from rises in the stock market. The new guarantee condition is as follows:

$$
V_{t} \geq \max \left(P_{t}, k \times \sup _{s \leq t} V_{s}\right),
$$

with $k$ an exogenous parameter lying between 0 and 1 (usually equal to 0.9 ). It means that the investor does not want to loose more than a given percentage of the maximum of his past portfolio values. This portfolio strategy belongs to CPPI strategies but associated to a stochastic floor given by $\max \left(P_{t}, k \times\right.$ $\left.\sup _{s \leq t} V_{s}\right)$.

- The multiple can vary according to market and portfolio dynamics. Prigent (2001) considers a more general exposure function $e_{t}=e\left(t, C_{t}\right)$ defined on $[0, T] \times \mathbb{R}^{+}$, positive and continuous, with conditions on $e(t, C)$ ensuring that the cushion always is positive. If the cushion is nil, then the exposure must be equal to 0 (i.e. $e(t, 0)=0$ ) and if the relative sizes of jumps $\frac{\Delta S}{S}$ have a lower bound $d$ (negative), then, for any $(t, C)$, we must have:

$$
e(t, C) \leq-\frac{1}{d} C .
$$

In what follows, we still assume a linear function of the cushion, but now the multiple is a random process depending for example on given state variables such as the risky asset volatility. We illustrate the determination of the conditional multiple as function of state variables, within the standard or generalized Johnson distributions. 


\subsection{Quantile hedging and conditional multiple}

\subsubsection{Quantile condition}

In what follows, as in Ben Ameur and Prigent (2014), we focus on local quantile condition. Consider the filtration $\left(\mathcal{F}_{t_{k}}\right)_{k}$ generated by the observations of the returns $\left(R_{t_{k}}\right)_{k}$. Consider a given sequence of thresholds $L_{t_{k-1}}>0$ (these thresholds can be fixed or variable). The local quantile condition is defined by:

$$
\mathbb{P}^{\mathcal{F}_{t_{k-1}}}\left[C_{t_{k}}>L_{t_{k-1}}\right] \geq(1-a),
$$

where $\mathbb{P}^{\mathcal{F}_{t_{k-1}}}$ denotes the conditional probability with respect to information $\mathcal{F}_{t_{k-1}}$.

Recall that the cushion value is determined from the following relation ${ }^{2}$

$$
C_{t_{k}}=C_{t_{k-1}} \times\left(1+m_{t_{k-1}} \times \frac{\Delta S_{t_{k}}}{S_{t_{k-1}}}\right) .
$$

The guarantee condition at any time $t_{k}$ is: $C_{t_{k}}>L_{t_{k-1}}$.

Case 1: $\left(C_{t_{k-1}}>0\right)$

$$
\begin{aligned}
& C_{t_{k}}>L_{t_{k-1}} \Longleftrightarrow 1+m_{t_{k-1}} \times \frac{\Delta S_{t_{k}}}{S_{t_{k-1}}}>\frac{L_{t_{k-1}}}{C_{t_{k-1}}}, \\
& C_{t_{k}}>L_{t_{k-1}} \Longleftrightarrow 1+m_{t_{k-1}} \times\left(R_{t_{k}}-1\right)>\frac{L_{t_{k-1}}}{C_{t_{k-1}}}, \\
& C_{t_{k}}>L_{t_{k-1}} \Longleftrightarrow 1+m_{t_{k-1}} \times\left[\exp \left[h_{t_{k}}^{-1}\left(\varepsilon_{t_{k}}\right) \sigma_{t_{k}}+\mu_{t_{k}}\right]-1\right]>\frac{L_{t_{k-1}}}{C_{t_{k-1}}} .
\end{aligned}
$$

Case 2: $\left(C_{t_{k-1}}<0\right)$. In this case, this is not a true insurance condition. Therefore, a possible strategy is to adopt the previous condition when the cushion is positive and to invest the whole portfolio value on the riskless asset, as soon as the cushion is negative. Therefore, at any time $t_{k-1}$, the value of the multiple $m_{t_{k-1}}$ must be chosen such that, at time $t_{k}$, the cushion value satisfies: $C_{t_{k}}>L_{t_{k-1}}$. Consequently, $m_{t_{k-1}}$ can be searched as the following function:

$$
m_{t_{k-1}}=m_{t_{k-1}}^{(1)} \times \mathbb{I}_{C_{t_{k-1}}>L_{t_{k-1}}}+m_{t_{k-1}}^{(2)} \times \mathbb{I}_{C_{t_{k-1}} \leq L_{t_{k-1}}},
$$

where both $m_{t_{k-1}}^{(1)}$ and $m_{t_{k-1}}^{(2)}$ are random variables which are $\mathcal{F}_{t_{k-1}}-$ measurable.

\subsubsection{Determination of the multiple}

In what follows, we search for explicit forms of the random variables $m_{t_{k-1}}^{(1)}$ and $m_{t_{k-1}}^{(2)}$. We assume that the funds dynamics corresponds to an EGARCH type model, defined by:

$$
R_{t_{k}}=\frac{S_{t_{k}}}{S_{t_{k-1}}}=\exp \left[Z_{t_{k}}\right] \text { with } Z_{t_{k}}=\mu_{t_{k}}+\sigma_{t_{k}} h_{t_{k}}^{-1}\left(\varepsilon_{t_{k}}\right),
$$

\footnotetext{
${ }^{2}$ Since we consider small time intervals, we set $r_{t_{k}}=0$.
} 
where $Z_{t_{k}}$ is solution of the system of the following autoregressive equations:

$$
\left\{\begin{array}{c}
Z_{t_{k}}=\alpha_{0}+\sum_{i=1}^{P} \alpha_{i} \times Z_{t_{k-i}}+\sigma_{t_{k}} \times \epsilon_{t_{k}}, \\
\Lambda\left(\sigma_{t_{k}}\right)=\beta+C_{0}\left(\epsilon_{t_{k-1}}\right)+C_{1}\left(\epsilon_{t_{k-1}}\right) \times \Lambda\left(\sigma_{t_{k-1}}\right),
\end{array}\right.
$$

where $\sigma_{t_{k}}$ denotes the volatility, the sequence $\left(\epsilon_{t_{k}}\right)_{k}$ is i.i.d. with common pdf $f>0$ and $\Lambda, C_{0}($.$) , and C_{1}($.$) are deterministic functions. The function \Lambda$ : $\mathbb{R}^{+} \rightarrow \mathbb{R}$ is assumed to be strictly increasing. We set

$$
\mu_{t_{k}}=\alpha_{0}+\sum_{i=1}^{P} \alpha_{i} \times Z_{t_{k-i}} .
$$

The information delivered by the observation of risk asset returns is generated by the $\left(\epsilon_{t_{1}}, \ldots, \epsilon_{t_{k-1}}\right)_{k}$. We have:

$$
\mathcal{F}_{t_{k-1}}=\sigma \text {-algebra }\left(\epsilon_{t_{1}}, \ldots, \epsilon_{t_{k-1}}\right) .
$$

Thus we deduce that the random variables $m_{t_{k-1}}^{(1)}$ and $m_{t_{k-1}}^{(2)}$ are deterministic functions of $\left(\epsilon_{t_{1}}, \ldots, \epsilon_{t_{k-1}}\right)$. Therefore, we have to search for a multiple $m_{t_{k}}$ which has the following form:

$$
\begin{gathered}
m_{t_{k-1}}=m^{(1)}\left(t_{k-1}, Z_{t_{1}}, \ldots, Z_{t_{k-1}}, \sigma_{t_{1}}, \ldots, \sigma_{t_{k}}\right) \times \mathbb{I}_{C_{t_{k-1}}>L_{t_{k-1}}} \\
\quad+m^{(2)}\left(t_{k-1}, Z_{t_{1}}, \ldots, Z_{t_{k-1}}, \sigma_{t_{1}}, \ldots, \sigma_{t_{k}}\right) \times \mathbb{I}_{C_{t_{k-1}} \leq L_{t_{k-1}}},
\end{gathered}
$$

where the parameter $L_{t_{k-1}}$ corresponds to a given threshold $\left(L_{t_{k-1}}>0\right)$.

Remark 7 Note that the random variables $Z_{t_{k-1}}$ are deterministic functions of the random vector $\left(\epsilon_{t_{1}}, \ldots, \epsilon_{t_{k-1}}\right)$. But, for a better financial explanation of the multiple, we explicitly introduce functions of vector $\left(Z_{t_{1}}, \ldots, Z_{t_{k-1}}\right)$ itself. This allows to analyze how the conditional multiple depends on both the volatility levels and on the past values of $Z$ related to returns, which are here the state variables.

In order to determine the conditional multiple, two cases have to be distinguished $^{3}$ :

- The cushion at time $t_{k-1}$ is non negative: $C_{t_{k-1}}>L_{t_{k-1}}$.

- The cushion at time $t_{k-1}$ satisfies: $0<C_{t_{k-1}}<L_{t_{k-1}}$.

Consider the conditional $\operatorname{cdf} F^{\mathcal{F}_{t_{k-1}}}$ of $Z_{t_{k-1}}$.

We obtain the following results ${ }^{4}$ (the cushion $C_{t_{k-1}}$ at time $t_{k-1}$ is assumed to be strictly positive (otherwise, we stop investing in the risky asset).

\footnotetext{
${ }^{3}$ Since we assume that the pdf of the random variables $\epsilon$ is non negative, the probability of the event $C_{t_{k-1}}=L_{t_{k-1}}$ is null.

${ }^{4}$ For the proofs of the results that follow, see Appendix.
} 
Consider at first the case where the threshold $L_{t_{k-1}}$ is arbitrary.

Proposition 8 When $C_{t_{k-1}}>L_{t_{k-1}}$, the quantile condition at time $t_{k-1}$ can be described as follows:

- i) If $\frac{\frac{L}{C_{t_{k-1}}}-1}{m_{t_{k-1}}}+1<0$, then $\mathbb{P}^{\mathcal{F}_{t_{k-1}}}\left[C_{t_{k}}>L_{t_{k-1}}\right]=1$.

- ii) If $C_{t_{k-1}}>L_{t_{k-1}}$ and $\frac{\frac{L_{t_{k-1}}}{C_{t_{k-1}}}-1}{m_{t_{k-1}}}+1>0$, then:

- a) If $\exp \left[h_{t_{k}}^{-1}\left(q_{(a, T)}^{\varepsilon}\right) \sigma_{t_{k}}+\mu_{t_{k}}\right]<1$, then

If $\frac{L_{t_{k-1}}}{C_{t_{k-1}}}<\exp \left[h_{t_{k}}^{-1}\left(q_{(a, T)}^{\varepsilon}\right) \sigma_{t_{k}}+\mu_{t_{k}}\right]$, then $m_{t_{k-1}}$ must satisfy the following constraint:

$$
m_{t_{k-1}} \leq \frac{\frac{L_{t_{k-1}}}{C_{t_{k-1}}}-1}{\exp \left[h_{t_{k}}^{-1}\left(q_{(a, T)}^{\varepsilon}\right) \sigma_{t_{k}}+\mu_{t_{k}}\right]-1} .
$$

If $\frac{L_{t_{k-1}}}{C_{t_{k-1}}}>\exp \left[h_{t_{k}}^{-1}\left(q_{(a, T)}^{\varepsilon}\right) \sigma_{t_{k}}+\mu_{t_{k}}\right]$, then the previous constraint must be satisfied but it also implies that $m_{t_{k-1}}<1$ (the upper bound is strictly lower than 1).

- b) If $\exp \left[h_{t_{k}}^{-1}\left(q_{(a, T)}^{\varepsilon}\right) \sigma_{t_{k}}+\mu_{t_{k}}\right]>1$, then there is no constraint on $m_{t_{k-1}}$.

The previous proposition shows that, if the multiple is very small, the quantile condition is automatically satisfied (see assertion i). If the value of the multiple is higher (see assertion ii), then, if the market value $S$ decreases (case ii-a), the multiple should be bounded and even smaller than 1 if the previous cushion value is not high. In this latter case, we have to stop the investment in $S$. If the value of return is sufficiently high (case ii-b), there is no constraint on the multiple.

Proposition 9 When $0<C_{t_{k-1}}<L_{t_{k-1}}$, the quantile condition at time $t_{k-1}$ is satisfied according to the following conditions:

- i) If $\exp \left[h_{t_{k}}^{-1}\left(q_{(a, T)}^{\varepsilon}\right) \sigma_{t_{k}}+\mu_{t_{k}}\right]<1$, there is no positive solution for $m_{t_{k-1}}$.

- ii) If $\exp \left[h_{t_{k}}^{-1}\left(q_{(a, T)}^{\varepsilon}\right) \sigma_{t_{k}}+\mu_{t_{k}}\right]>1$ :

on $m_{t_{k-1}}$.

$$
\text { If } \frac{L_{t_{k-1}}}{C_{t_{k-1}}}<\exp \left[h_{t_{k}}^{-1}\left(q_{(a, T)}^{\varepsilon}\right) \sigma_{t_{k}}+\mu_{t_{k}}\right] \text {, then there is no constraint }
$$

following constraint:

$$
\text { If } \frac{L_{t_{k-1}}}{C_{t_{k-1}}}>\exp \left[h_{t_{k}}^{-1}\left(q_{(a, T)}^{\varepsilon}\right) \sigma_{t_{k}}+\mu_{t_{k}}\right] \text {, then } m_{t_{k-1}} \text { must satisfy the }
$$

$$
m_{t_{k-1}} \geq \frac{\frac{L_{t_{k-1}}}{C_{t_{k-1}}}-1}{\exp \left[h_{t_{k}}^{-1}\left(q_{(a, T)}^{\varepsilon}\right) \sigma_{t_{k}}+\mu_{t_{k}}\right]-1} .
$$


The previous result corresponds to the search for a minimum portfolio performance. In fact, the threshold $L_{t_{k-1}}$ being positive, we seek permanently to maintain the cushion above a minimum positive value, even if we are more exposed to the risk of fluctuation in the risky asset. Thus, if the initial value of the cushion is below the threshold $L_{t_{k-1}}$ and the return of the risky asset is not sufficiently high, there is no solution. If, on the contrary, the return of the risky asset is relatively high, there is no constraint on the multiple. For an intermediate value of the risky asset return, we must compensate with sufficient exposure. It means that the multiple must be higher than a minimum value.

Remark 10 As soon as the cushion is positive at time $t_{k-1}$, the choice of the multiple is very flexible. Therfore, under the quantile condition at time $t_{k-1}$, other conditions on the multiple can be introduced to better benefit from market variations. As soon as the cushion is negative (small probability), the quantile condition generally does no longer hold, except for small values of $(1-\epsilon)$. However, in this latter case, this does not correspond to a true insurance condition. Consequently, a practical strategy is to use previous bounds on the multiple when the cushion is positive and to invest the whole portfolio value on the riskless asset, as soon as the cushion is negative.

\subsection{Expected shortfall conditions}

We consider now the local Expected Shortfall (ES or CVaR) criterion. Recall that the VaR measure is the potential loss at a given probability level $a$ but VaR does not provide any information about the severity of loss by which it is exceeded. To remedy this issue, we can alternatively introduce the expected shortfall since it is the expectation of losses exceeding the VaR, knowing that the loss is beyond the VaR. In such a framework, first we determine the multiple $m_{t_{k-1}}$ with the VaR condition (see previous propositions):

$$
\left.\mathbb{P}\left[C_{t_{k}} \leq L_{t_{k-1}} \mid \mathcal{F}_{t_{k-1}} \cap C_{t_{k-1}}>0\right]=a, a \epsilon\right] 0,1[.
$$

Second, we minimize the expected shortfall defined by:

$$
L_{t_{k-1}}-\mathbb{E}\left[C_{t_{k}} \mid \mathcal{F}_{t_{k-1}} \cap C_{t_{k-1}}>0 \cap C_{t_{k}} \leq L_{t_{k-1}}\right] .
$$

Such approach is detailed in what follows.

Step 1: We define the set $M_{t_{k-1}}\left(a, L_{t_{k-1}}\right)$ corresponding to the previous local quantile condition.

Step 2: Then, we calculate the local expected shortfall. Let us consider the case $L_{t_{k-1}} \geq 0$. Recall that:

$$
C_{t_{k}}=C_{t_{k-1}}\left[1+m_{t_{k-1}}\left(R_{t_{k}}-1\right)\right] .
$$

Therefore we get:

$$
\mathbb{E}\left[L_{t_{k-1}}-C_{t_{k}} \mid C_{t_{k-1}}>0 \cap \mathcal{F}_{t_{k-1}} \cap C_{t_{k}}<L_{t_{k-1}}\right]=
$$




$$
\frac{\mathbb{E}\left[\left(L_{t_{k-1}}-C_{t_{k}}\right) \mathbb{I}_{C_{t_{k}}<L_{t_{k-1}}} \mid C_{t_{k-1}}>0 \cap \mathcal{F}_{t_{k-1}}\right]}{\mathbb{P}\left[C_{t_{k}}<L_{t_{k-1}} \mid C_{t_{k-1}}>0 \cap \mathcal{F}_{t_{k-1}}\right]},
$$

where $\mathbb{I}$ is the Heavyside function that indicates if $C_{t_{k}}$ is less than $L_{t_{k-1}}$. Denote $\Phi_{L}\left(m_{t_{k-1}}\right)$ by:

$$
\Phi_{L}\left(m_{t_{k-1}}\right)=\frac{\mathbb{E}\left[\left(L_{t_{k-1}}-C_{t_{k}}\right) \mathbb{I}_{C_{t_{k}}<L_{t_{k-1}}} \mid C_{t_{k-1}}>0 \cap \mathcal{F}_{t_{k-1}}\right]}{\mathbb{P}\left[C_{t_{k}}<L_{t_{k-1}} \mid C_{t_{k-1}}>0 \cap \mathcal{F}_{t_{k-1}}\right]} .
$$

Determination of $\Phi_{L}\left(m_{t_{k-1}}\right)$ :

$\left.\overline{\text { Since } C_{t_{k}}=C_{t_{k-1}}\left[1+m_{t_{k-1}}\right.}\left(R_{t_{k}}-1\right)\right]$ and $C_{t_{k-1}}>0$, then we get the following characterization:

$$
C_{t_{k}}<L_{t_{k-1}} \Leftrightarrow\left[1+m_{t_{k-1}}\left(R_{t_{k}}-1\right)\right]<\frac{L_{t_{k-1}}}{C_{t_{k-1}}},
$$

which is equivalent to:

$$
R_{t_{k}}<\frac{\frac{L_{t_{k-1}}}{C_{t_{k-1}}}-1}{m_{t_{k-1}}}+1
$$

and also to

$$
\varepsilon_{t_{k}}<h_{t_{k}}\left(\frac{1}{\sigma_{t_{k}}} \ln \left(\frac{\frac{L_{t_{k-1}}}{C_{t_{k-1}}}-1}{m_{t_{k-1}}}+1\right)-\frac{\mu_{t_{k}}}{\sigma_{t_{k}}}\right) .
$$

Denote $l_{L}\left(m_{t_{k-1}}\right)=h_{t_{k}}\left(\frac{1}{\sigma_{t_{k}}} \ln \left(\frac{\frac{L_{t_{k-1}}}{C_{t_{k-1}}}-1}{m_{t_{k-1}}}+1\right)-\frac{\mu_{t_{k}}}{\sigma_{t_{k}}}\right)$. Then, the conditional expectation

$$
\mathbb{E}\left[L_{t_{k-1}}-C_{t_{k}} \mid \mathcal{F}_{t_{k-1}} \cap C_{t_{k-1}}>0 \cap C_{t_{k}}<L_{t_{k-1}}\right]
$$

is equal to

$$
L_{t_{k-1}}-\frac{C_{t_{k-1}} \int_{-\infty}^{l_{L}\left(m_{t_{k-1}}\right)}\left[1+m_{t_{k-1}}\left(\exp \left[\sigma_{t_{k}} h_{t_{k}}^{-1}(u)+\mu_{t_{k}}\right]-1\right)\right] \times f_{\varepsilon_{t_{k}}}(u) d u}{N\left(l_{L}\left(m_{t_{k-1}}\right)\right.} .
$$

Since $\varepsilon_{t_{k}}$ is Gaussian, we have:

$$
f_{\varepsilon_{t_{k}}}(u)=\frac{1}{\sqrt{2 \pi}} \exp \left[-\frac{u^{2}}{2}\right], N(u)=\int_{-\infty}^{u} f_{\varepsilon_{t_{k}}}(v) d v .
$$

Then, we have:

$$
\begin{gathered}
\Phi_{L}\left(m_{t_{k-1}}\right)=L_{t_{k-1}}- \\
C_{t_{k-1}}\left(1-m_{t_{k-1}}\right) \frac{m_{t_{k-1}} C_{t_{k-1}} \int_{-\infty}^{l_{L}\left(m_{t_{k-1}}\right)}\left(\exp \left[\mu_{t_{k}}+\sigma_{t_{k}} h_{t_{k}}^{-1}(u)\right]\right) \frac{1}{\sqrt{2 \pi}} \exp \left(\frac{-u^{2}}{2}\right) d u}{N\left(l_{L}\left(m_{t_{k-1}}\right)\right.}
\end{gathered}
$$


Therefore:

$$
\Phi\left(m_{t_{k-1}}\right)=L_{t_{k-1}}-C_{t_{k-1}}\left[\left(1-m_{t_{k-1}}\right)+\frac{m_{t_{k-1}}}{N\left(l_{L}\left(m_{t_{k-1}}\right)\right)} \times I_{L}\left(m_{t_{k-1}}\right)\right],
$$

with

$$
I_{L}=\frac{1}{\sqrt{2 \pi}} \int_{-\infty}^{l_{L}\left(m_{t_{k-1}}\right)} \exp \left[\mu_{t_{k}}+\sigma_{t_{k}} h_{t_{k}}^{-1}(u)\right] \exp \left(\frac{-u^{2}}{2}\right) d u .
$$

Note that, for $L_{t_{k-1}}=0$, we get:

$$
l_{0}\left(m_{t_{k-1}}\right)=h_{t_{k}}\left(\frac{1}{\sigma_{t_{k}}} \ln \left(\frac{-1}{m_{t_{k-1}}}+1\right)-\frac{\mu_{t_{k}}}{\sigma_{t_{k}}}\right) .
$$

where $l_{0}\left(m_{t_{k-1}}\right)$ does not depend on the cushion value $C_{t_{k-1}}$ at time $t_{k-1}$, and we get:

$$
\Phi_{0}\left(m_{t_{k-1}}\right)=-C_{t_{k-1}}\left[\left(1-m_{t_{k-1}}\right)+\frac{m_{t_{k-1}}}{N\left(l_{0}\left(m_{t_{k-1}}\right)\right)} \times I_{0}\right],
$$

with

$$
I_{0}=\frac{1}{\sqrt{2 \pi}} \int_{-\infty}^{l_{0}\left(m_{t_{k-1}}\right)} \exp \left[\mu_{t_{k}}+\sigma_{t_{k}} h_{t_{k}}^{-1}(u)\right] \exp \left(\frac{-u^{2}}{2}\right) d u
$$

Finally, we minimize the local expected shortfall for conditional multiples satisfying the local quantile condition (i.e. $m_{t_{k-1}} \in M_{t_{k-1}}\left(a, L_{t_{k-1}}\right)$ ).

$$
\begin{gathered}
\widetilde{\mathbb{E S}}_{\alpha}\left[C_{t_{k}}\right]= \\
\operatorname{Min}_{\left\{m_{t_{k-1}} \in M_{t_{k-1}}\left(a, L_{t_{k-1}}\right)\right\}}\left(L_{t_{k-1}}-\mathbb{E}\left[C_{t_{k}} \mid \mathcal{F}_{t_{k-1}} \cap C_{t_{k-1}}>0 \cap C_{t_{k}} \leq L_{t_{k-1}}\right]\right) .
\end{gathered}
$$

The expected shortfall is minimized for values of the multiple variable $m_{t_{k-1}}^{*}$ which lie between the two bounds of the multiple variable $m_{t_{k-1}}$ and satisfy the VaR condition. 


\section{Empirical illustrations}

\subsection{Estimation of hedge funds returns by Johnson system}

We determine the family and estimate the parameters of Johnson distributions for the 10 sub-indices of hedge funds with three methods, namely the method of moments using the algorithm of Hill et al. (1976), the method of quantile of Wheeler (1980) and the other method of quantile considered by Slifker and Shapiro (1980). Since we have three different estimation methods, we perform a fit test of $\chi^{2}$ to differentiate them.

Our study is conducted on the Credit Suisse Hedge Fund Index provided by Credit Suisse. These indexes provide the monthly returns of the ten main families of alternative strategies identified by this data provider. These indices are Convertible Arbitrage (CA), Dedicated Short Bias (DSB), Emerging Markets (EM), Equity Market Neutral (EMN), Event Driven (ED), Fixed Income Arbitrage (FIA), Global Macro (GM), Long / Short Equity (LSE), Managed Futures (MF) and Multi-Strategy (MF).

The global index constructed from the monostrategy indices is used to represent all hedge fund strategies. These indices are constructed using the TASS database which includes more than 3000 U.S. and offshore hedge funds. The characteristic of these indices is that they are known by the transparency guaranteed by Credit Swiss in their communication and construction mode.

These indices include only funds having at least $\$ 10$ million under management and providing audited financial reports. After this filtering, 400 funds on average are retained. The procedure for selecting funds. is renewed quarterly to update calculations. In fact, if a fund no longer satisfies expectations of financial reporting, it is systematically excluded. As to the classification of funds by major styles of strategy, it is realized on the basis of declarations of the managers. Funds are then weighted by their capitalization and presented net of commissions.

The study of indices is conducted on the period from December 1994 until December 2013, which gives us the possibility to work on 240 monthly observations. This period covers a very significant number of financial crises. 
Table 1: Distribution type and Johnson parameters estimated by the method of moments

\begin{tabular}{lrrrcc}
\hline & \multicolumn{1}{c}{$\gamma$} & \multicolumn{1}{c}{$\delta$} & \multicolumn{1}{c}{$\lambda$} & Type \\
\hline Convertible Arbitrage & 0.8394 & 1.2845 & 0.0178 & 0.0231 & $\mathrm{SU}$ \\
Dedicated Short Bias & -1.5101 & 2.5148 & -0.0674 & 0.0925 & $\mathrm{SU}$ \\
Event Driven & 1.2321 & 1.4236 & 0.0288 & 0.0168 & $\mathrm{SU}$ \\
Emerging Markets & 0.4364 & 1.4486 & 0.0283 & 0.0554 & $\mathrm{SU}$ \\
Equity Market Neutral & 0.4745 & 1.4760 & 0.0322 & 0.0617 & $\mathrm{SU}$ \\
Fixed Income Arbitrage & 1.8461 & 1.3189 & 0.0259 & 0.0084 & $\mathrm{SU}$ \\
Global Macro & -0.0221 & 1.4753 & 0.0087 & 0.0312 & $\mathrm{SU}$ \\
Long/Short Equity & -0.0259 & 1.6319 & 0.0071 & 0.0443 & $\mathrm{SU}$ \\
Managed Futures & 0 & 1 & 0.0333 & 0.0047 & $\mathrm{SN}$ \\
Multi-Strategy & 0.9981 & 1.7174 & 0.0235 & 0.0231 & $\mathrm{SU}$ \\
\hline
\end{tabular}

Table 2: Distribution type and Johnson parameters estimated by the method of Wheeler (1980)

\begin{tabular}{lrrrcc}
\hline & $\gamma$ & \multicolumn{1}{c}{$\delta$} & \multicolumn{1}{c}{$\xi$} & \multicolumn{1}{c}{$\lambda$} & Type \\
\hline Convertible Arbitrage & 0.2850 & 0.9563 & 0.0128 & 0.0116 & $\mathrm{SU}$ \\
Dedicated Short Bias & 0 & 4.8091 & -0.2286 & 0.2197 & $\mathrm{SL}$ \\
Event Driven & 0.6080 & 1.3611 & 0.0202 & 0.0201 & $\mathrm{SU}$ \\
Emerging Markets & 0.4059 & 1.1549 & 0.0273 & 0.0382 & $\mathrm{SU}$ \\
Equity Market Neutral & 0.3987 & 1.1663 & 0.0291 & 0.0439 & $\mathrm{SU}$ \\
Fixed Income Arbitrage & 0.3502 & 1.1513 & 0.0099 & 0.0100 & $\mathrm{SU}$ \\
Global Macro & -0.0087 & 1.1211 & 0.0093 & 0.0188 & $\mathrm{SU}$ \\
Long/Short Equity & -0.0908 & 1.5549 & 0.0052 & 0.0402 & $\mathrm{SU}$ \\
Managed Futures & 0 & 1 & 0.0041 & 0.0325 & $\mathrm{SN}$ \\
Multi-Strategy & 0.3350 & 1.0656 & 0.0133 & 0.0125 & $\mathrm{SU}$ \\
\hline
\end{tabular}


Table 3: Distribution type and Johnson parameters estimated by the method of Slifker and Shapiro (1980)

\begin{tabular}{lrcrcr}
\hline & $\gamma$ & $\delta$ & $\xi$ & \multicolumn{1}{c}{$\lambda$} & Type \\
\hline Convertible Arbitrage & 0.2558 & 0.9207 & 0.0125 & 0.0116 & $\mathrm{SU}$ \\
Dedicated Short Bias & -1.9022 & 1.4407 & -0.1321 & 0.0683 & $\mathrm{SL}$ \\
Event Driven & 0.7837 & 1.5433 & 0.0220 & 0.0219 & $\mathrm{SU}$ \\
Emerging Markets & 0.2825 & 1.3488 & 0.0213 & 0.0468 & $\mathrm{SU}$ \\
Equity Market Neutral & 0.2574 & 1.3361 & 0.0210 & 0.0496 & $\mathrm{SU}$ \\
Fixed Income Arbitrage & 0.2444 & 0.9018 & 0.0090 & 0.0074 & $\mathrm{SU}$ \\
Global Macro & -0.0045 & 0.9383 & 0.0096 & 0.0140 & $\mathrm{SU}$ \\
Long/Short Equity & -0.0434 & 1.5932 & 0.0070 & 0.0407 & $\mathrm{SU}$ \\
Managed Futures & -0.0141 & 2.4468 & 0.0045 & 0.1308 & $\mathrm{SU}$ \\
Multi-Strategy & 0.3745 & 1.2326 & 0.0139 & 0.0157 & $\mathrm{SU}$ \\
\hline
\end{tabular}

Tables (1), (2) and (3) show that the distribution type and parameter values may vary depending on the estimation method that is used. Indeed, for Dedicated Short Bias Index, according to the method of moments we find that the distribution is log-normal $(S L)$ while with the method of Wheeler (1980) and the method of Slifker and Shapiro (1980), we find that the distribution is unbounded $(S U)$. We also find that most of hedge fund returns have $S_{U}$ distribution. In order to choose the best method that corresponds to each index, we use the $\chi^{2}$ adequacy test. With this test, we retain the method that minimizes the value of $\chi^{2}$.

Table 4: Values of $\chi^{2}$ test according to different estimation methods

\begin{tabular}{lrrr}
\hline & Moments & Wheeler & Slifker \& Shapiro \\
\hline Convertible Arbitrage & 15.7779 & 1.5259 & 1.2867 \\
Dedicated Short Bias & 7.1041 & 16.2136 & 15.9684 \\
Event Driven & 13.1077 & 6.3583 & 7.9988 \\
Emerging Markets & 6.3238 & 10.2190 & 11.7220 \\
Equity Market Neutral & 5.8309 & 10.0031 & 11.8772 \\
Fixed Income Arbitrage & 59.0119 & 2.3502 & 2.5957 \\
Global Macro & 16.0726 & 4.7018 & 2.1119 \\
Long/Short Equity & 4.0751 & 2.4113 & 2.5970 \\
Managed Futures & 3.3392 & 3.3392 & 3.0278 \\
Multi-Strategy & 6.8135 & 2.6522 & 0.5765 \\
\hline
\end{tabular}

Table (4) shows that to each index corresponds a particular estimation method. 
In order to ensure the effectiveness of the Johnson transformation, we transform the series of returns of Multi-Strategy index to a series of random variables the normality of which we test (see Equations 1 and 2).
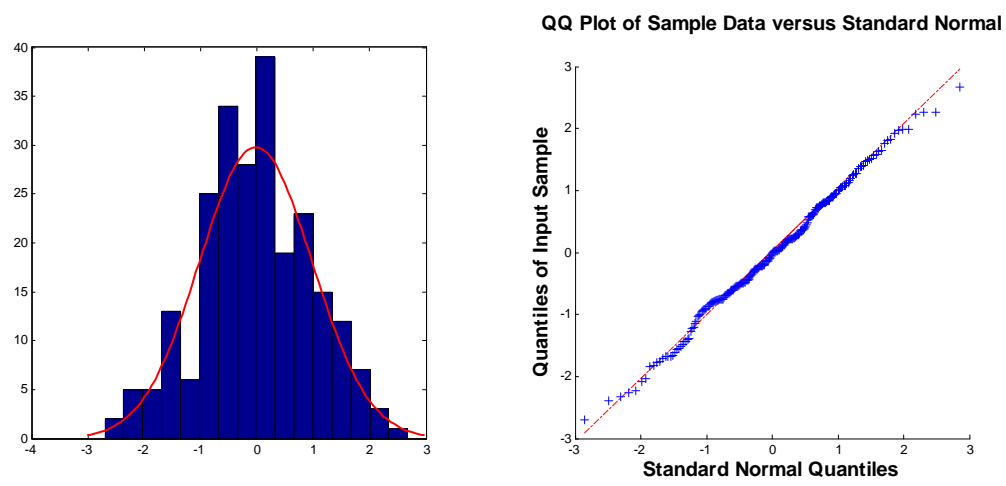

Figure 1: Histogram and QQ plot of transformed Multi Strategy index

Table 5: Statistical characteristics of the series of variables obtained by Johnson transformation

\begin{tabular}{|l|c|}
\hline \multicolumn{2}{|l|}{ Four first moments } \\
\hline Mean & -0.0206 \\
Standard deviation & 0.9991 \\
Skewness & 0.0037 \\
kurtosis & 2.8759 \\
\hline Kolmogorov-Smirnov test \\
\hline Normality & yes \\
p-value & 0.6987 \\
\hline Jarque-Bera test \\
\hline Normality & yes \\
p-value & 0,5000 \\
\hline
\end{tabular}

Figure (1) and Table (5) confirm the normality of distribution of random variables obtained by the Johnson transformation. This is in favor of the proposed approach based on Johnson distributions. 


\subsection{CPPI portfolio payoffs under quantile criterion}

To study the CPPI method with conditional multiple within Johnson distributions and temporal dependence of financial series, we choose the Multi Strategy

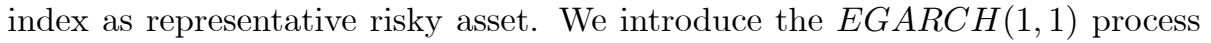
with Johnson type innovation to model the conditional volatility of log-returns. In what follows, we illustrate empirically our theoretical results. We simulate 1000 paths of CPPI portfolio values using conditional quantile criterion. The initial values of the portfolio and the price of the risky asset are equal to 100 . The period of the investment corresponds to 5 years and the guarantee rate required at maturity is $100 \%$ of the initial capital. We plot both the cdf of portfolio returns and the cdf of the ratio of portfolio values for a threshold corresponding to $L_{t_{k-1}}=0.75 C_{t_{k-1}}$.
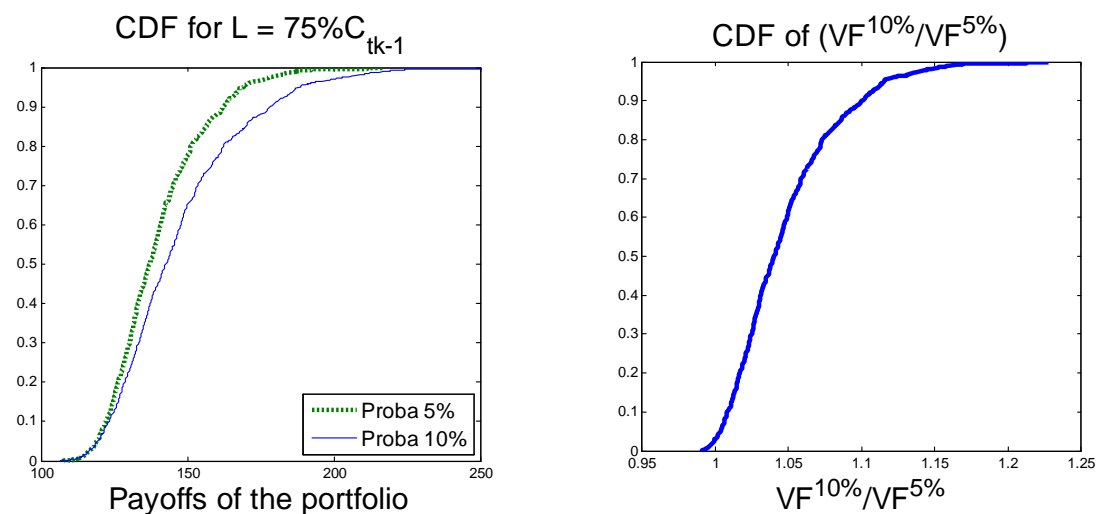

Figure 2: Distribution functions of final payments and ratios of CPPI portfolios with variable multiple under quantile criterion and different levels of loss probabilty

Figure (2) shows that, for a given threshold $L$, the portfolio with an acceptable loss probability of $10 \%$ stochastically dominates (approximately) at the first order the portfolio with an acceptable loss probability of $5 \%$. Indeed, portfolio associated to $5 \%$ can dominate the portfolio associated to $10 \%$ only for significant bearish market. This can be explained by the fact that the more risk averse the investor, the higher the proportion of his investment he wants to guarantee. This result is mainly due to the multiple values used in portfolio management. Indeed, for portfolios of $5 \%$, multiples are lower than those of $10 \%$. This leads to lower exposure to risk. We also note that for both levels of probability, the value of the initial investment is guaranteed in both cases. This is due to the method of choice of multiple. 


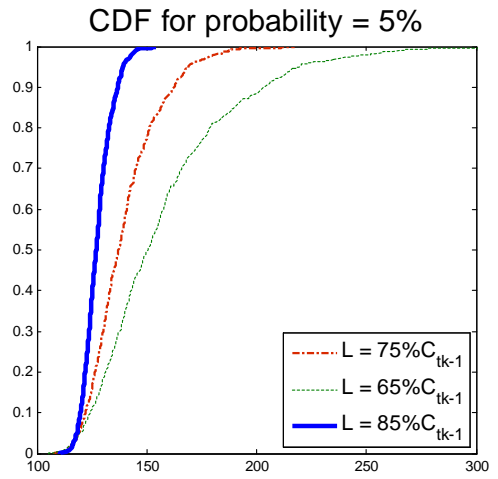

Payoffs of the portfolio

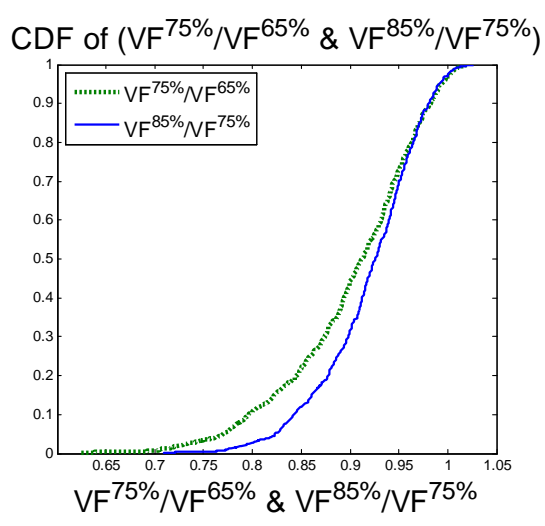

Figure 3: Distribution functions of CPPI portfolio payoffs with conditionnal multiple under quantile criterion and different levels of threshold

Figure (3) illustrates that, for a given probability level, the final value of the portfolio depends on the threshold $L$. The portfolio with a threshold $L_{t_{k-1}}=$ $65 \% C_{t_{k-1}}$ dominates the two portfolios with thresholds $75 \%$ of $C_{t_{k-1}}$ and $85 \%$ of $C_{t_{k-1}}$ for bullish market. On the contrary, it is dominated by the two portfolios for bearish market. The portfolio with threshold $L_{t_{k-1}}=75 \% C_{t_{k-1}}$ dominates the portfolio of threshold $65 \%$ of $C_{t_{k-1}}$ if the market fluctuation is moderate. This shows that an increase in the threshold level allows to increase probability to recover the initial investment but reduces it for bullish market. This seems logical because, to keep past performance, we must be exposed to less risk. This falls well within the framework of portfolio insurance. We also note that all portfolios were able to recover the initial investment. This illustrates the robustness of the method with CPPI multiple variable that takes account both of the conditional volatility of the risky asset and also the level of risk aversion of the investor.

\subsection{Payoff of the CPPI portfolio under expected shortfall criterion}

In the previous section, we have proved that the multiple chosen according to the expected shortfall criterion can be obtained by minimizing the function $\Phi_{L}\left(m_{t_{k-1}}\right)$. This function is the conditional expectation of the difference between the threshold value $L_{t_{k-1}}$ and the cushion $C_{t_{k}}$. In what follows, we present the different values of multiple under local expected shortfall criterion.

Recall that, under this criterion, the minimization problem corresponds to:

$$
\mathbb{E}\left[L_{t_{k-1}}-C_{t_{k}} \mid C_{t_{k-1}}>0 \cap \mathcal{F}_{t_{k-1}} \cap C_{t_{k}}<L_{t_{k-1}}\right] .
$$

and the function to minimize is given by: 


$$
\Phi_{L}\left(m_{t_{k-1}}\right)=L_{t_{k-1}}-C_{t_{k-1}}\left[\left(1-m_{t_{k-1}}\right)+\frac{m_{t_{k-1}}}{N\left(l_{L}\left(m_{t_{k-1}}\right)\right)} \times I_{L}\left(m_{t_{k-1}}\right)\right],
$$

We still choose the threshold value $L_{t_{k-1}}$ as a percentage $P$ of cushion $C_{t_{k-1}}$. This implies that we want to keep a percentage of the final realized performance. We study the three possible cases fo $P: P=0$ (in this case $L_{t_{k-1}}=0$ ), $P \leq 1$ and $P>1$. To determine the multiple that minimizes the function $\Phi_{L}\left(m_{t_{k-1}}\right)$, we set an interval $[1,10]$ in which can vary possible values of multiple. We find that, if $P \leq 1$, the multiple often takes the value of the upper bound of the interval and even by changing the boundaries of this interval, the multiple always takes the value of the upper bound. Furthermore, by putting $P>1$, we obtain different values of multiple. To determine the effect of $L_{t_{k-1}}$ on the choice of multiple, we choose three different values of $P$ are: $110 \%, 115 \%$ and $120 \%$. We simulate 1000 paths of CPPI portfolio values with a variable multiple selected according to expected shortfall criterion. The initial values of the portfolio and the price of the risky asset are equal to 100 .
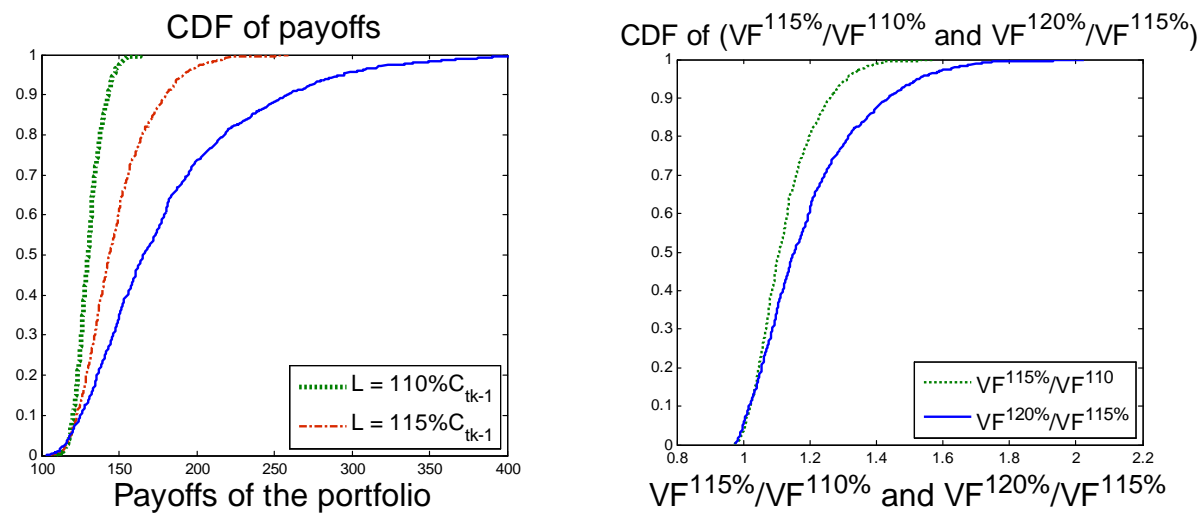

Figure 4: Cdf of CPPI portfolios with variable multiple under expected shortfall criterion with different values of $\mathrm{L}$

Figure (4) shows that the portfolio with $L_{t_{k-1}}=120 \% C_{t_{k-1}}$ dominates portfolios with $L_{t_{k-1}}=110 \% C_{t_{k-1}}$ and $L_{t_{k-1}}=115 \% C_{t_{k-1}}$ when the market rises. However, it is dominated by these two portfolios for bearish market. We explain these results by the fact that if we want to get better performance than the last realized performance, we must increase our risk exposure by using higher values of multiple. We also find that, in all cases, the probability of losing the initial investment is equal to zero. Even if we are much exposed to risk, at maturity we can reimburse the full amount initially invested. This also proves the robustness of the method we use to determine the conditional multiple. 


\subsection{Comparison between final values of the CPPI accord- ing to the method of choice of multiple}

To evaluate the different methods of multiple choice, we make a comparison between the three methods that we have presented: fixed multiple, multiple with quantile criterion and multiple with expected shortfall criterion.
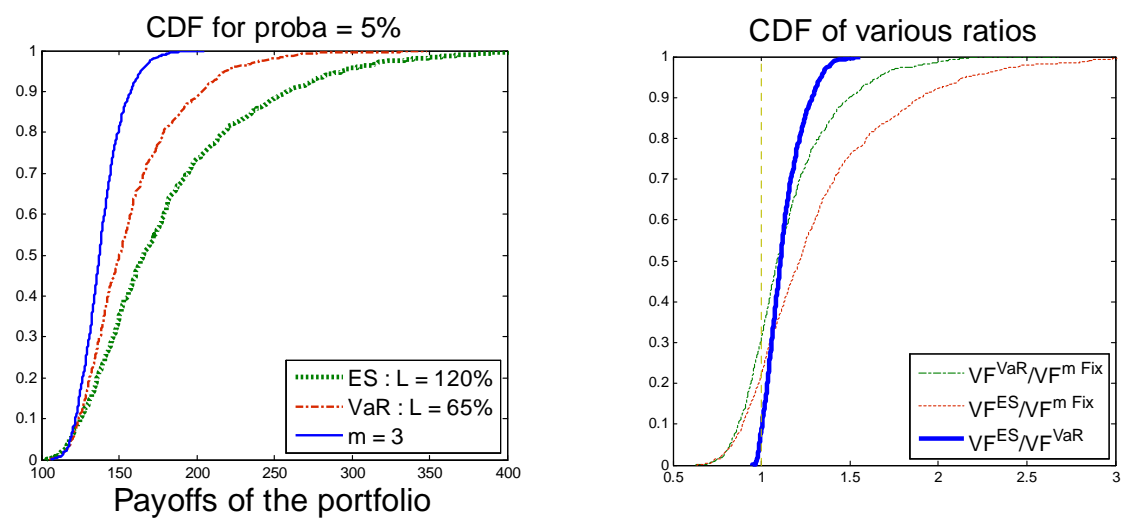

Figure 5: Cdf of CPPI portfolio payoffs under three methods to choose the multiple

Figure (5) shows that the portfolio with expected shortfall criterion dominates the portfolio with quantile criterion and the portfolio with fixed multiple. In fact, we have a probability equal to $90 \%$ that the final portfolio value with expected shortfall criterion is higher than the portfolio with quantile criterion and a probability equal to $75 \%$ that it is higher than the portfolio with fixed multiple. This shows that, even if the investor is exposed to more risk, he can achieve a good performance while ensuring a better hedge against downside risk by choosing a multiple variable that minimizes the expectation of the difference between the value of threshold and that of the cushion. We also find that the portfolio with quantile criterion dominates portfolio with fixed multiple when the market is bearish. We have a probability equal to $80 \%$ that the final value of the quantile portfolio is higher than the portfolio with a fixed multiple. When the market increases moderately, the portfolio associated to a fixed multiple dominates the portfolio corresponding to quantile criterion. From this comparison, we can conclude that the method based on a variable multiple can provide better performance and hedging against the risk, whatever the evolution of the risky asset. This is explained by the introduction of the conditional volatility estimated by $\operatorname{EGARCH}(1,1)$ with a distribution of innovation that takes the higher order moments into account. 


\section{Conclusion}

We have examined CPPI strategy on hedge funds. Due to the non normality of their returns, we have introduced Johnson distributions. We have also assumed that rebalancing times are discrete, which corresponds to actual practice. We have provided upper bounds on conditional multiple, by introducing gap risk controlled by means of both quantile and expected shortfall criteria. The upper bound of the multiple is a function of the given probability level, of the threshold $L$ and of the conditional volatility of logarithmic returns of risky asset. This latter one is estimated from the $\operatorname{EGARCH}(1,1)$ with innovations that follow Johnson distribution $S U$. Results show that by taking these criteria into account, we can provide a better portfolio insurance while having a better ability to profit from market increases. 


\section{References}

[1] Ackerman, C., McEnally, R. and D. Ravenscraft (1999). The performance of hedge funds: risk, return and incentives. Journal of Finance, 54, 833-874.

[2] Agarwal, V. and N.Y. Naik (2004). Risks and portfolio decisions involving hedge funds. Review of Financial Studies, 17, 63-98.

[3] Bacmann, J.-F. and S. Scholz (2003). Alternative performance measures for hedge funds. AIMA Journal, June.

[4] Balder, S., Brandl, S. and A. Mahayni. (2009): Effectiveness of CPPI strategies under discrete-time trading. Journal of Economic Dynamics and Control, 33, 204-220.

[5] Ben Ameur, H. and J.-L. Prigent (2014). Portfolio Insurance: Gap risk under conditional multiples. European Journal of Operational Research, 236, 238-253.

[6] Bertrand, P. and J-L. Prigent (2002). Portfolio insurance: the extreme value approach to the CPPI method. Finance, 23, 69-86.

[7] Bertrand, P. and J-L. Prigent (2011). Omega performance measure and portfolio insurance. Journal of Banking and Finance, 35, 1811-1823.

[8] Black, F. and R. Jones (1987). Simplifying portfolio insurance. Journal of Portfolio Management, 14, 48-51.

[9] Black, F. and A.R. Perold (1992). Theory of constant proportion portfolio insurance. The Journal of Economics, Dynamics and Control, 16, 403-426.

[10] Black, F., and R. Rouhani (1989). Constant proportion portfolio insurance and the synthetic put option: A comparison. In Investment Management, ed. by Fabozzi F. J., Ballinger, Cambridge, Massachusetts.

[11] Brooks, C and H.M. Kat (2001). The Statistical Properties of Hedge Fund Index Returns and their Implications for Investors. Working Paper, ISMA Centre Discussion Papers In Finance.

[12] Brown, S.J., Goetzmann, W. N. and R. G. Ibbotson (1999). Offshore hedge funds: survival and performance: 1989-1995. Journal of Business, 72, 91119 .

[13] Caglayan, M. and F. Edwards (2001). Hedge fund performance and manager skill. Journal of Futures Markets, 21, 1003-1028.

[14] Ested, T. and M. Kritzman (1988). TIPP: insurance without complexity, Journal of Portfolio Management, 14, 38-42.

[15] Föllmer, H. and P. Leukert (1999). Quantile hedging, Finance and Stochastics, 3, 251-273. 
[16] Fung, W., and D. Hsieh. (1997). Empirical characteristics of dynamic trading strategies: The case of hedge funds. Review of Financial Studies, 10, 275-302.

[17] Hamidi, B., Jurczenko, E., and B. Maillet, (2009a). D'un multiple conditionnel en assurance de portefeuille : CaViaR pour les gestionnaires? University of Paris 1. Working paper CES 2009.33.

[18] Hamidi, B., Jurczenko, E., and B. Maillet, (2009b), "A CAViaR timevarying proportion portfolio insurance. Bankers, Markets and Investors 102, September-October, 4-21.

[19] Hamidi, B., Maillet, B. and J.L. Prigent, (2014). Dynamic autoregressive expectile for time-invariant portfolio protection strategies. To appear in Journal of Economics Dynamics and Control.

[20] Hill, I.D., Hill, R., and R.L. Holder (1976). Fitting Johnson curves by moments. Applied Statistics, AS99.

[21] Johnson, N.L. (1949). Systems of frequency curves generated by methods of translation. Biometrica, $58,547-558$.

[22] Longin, F. (1997). The treshold effect in expected volatility: A model based on asymmetric Information. Review of Financial Studies, 10, 837-869.

[23] Perold, A.R. (1986). Constant Proportion Portfolio Insurance. Harvard Business School, Working paper.

[24] Perold, A.R. and W. Sharpe, (1988). Dynamic strategies for asset allocations. Financial Analysts Journal, 44, 16-27.

[25] Poncet, P. and R. Portait (1997). Assurance de Portefeuille in Y. Simon ed. Encyclopédie des Marchés Financiers, Economica,140-141.

[26] Prigent, J.L. (2001). Assurance du portefeuille: analyse et extension de la méthode du coussin, Banque et Marchés, 51, 33-39.

[27] Roman E., Kopprash R. and E. Hakanoglu (1989). Constant Proportion Portfolio Insurance for fixed-income investment. Journal of Portfolio Management, 15, 58-66.

[28] Slifker, J.F. and S.S. Shapiro (1980). The Johnson system: selection and parameter estimation. Technometrics, 22, 239-246.

[29] Wheeler, R. E. (1980). Quantile estimators of Johnson curve parameters. Biometrika, 67, 725-728. 


\section{Appendix}

\section{Proofs of propositions 8 and 9}

In what follows, the cushion at time $t_{k-1}$ is strictly positive $\left(C_{t_{k-1}}>0\right)$.

Case 1: $C_{t_{k-1}}>L_{t_{k-1}}$.

The local quantile condition is the following:

$$
\mathbb{P}^{\mathcal{F}_{t_{k-1}}}\left[1+m_{t_{k-1}} \times \frac{\Delta S_{t_{k}}}{S_{t_{k-1}}}>\frac{L_{t_{k-1}}}{C_{t_{k-1}}}\right] \geq(1-a),
$$

which is equivalent to:

$$
\mathbb{P}^{\mathcal{F}_{t_{k-1}}}\left[m_{t_{k-1}}\left(R_{t_{k}}-1\right)>\frac{L_{t_{k-1}}}{C_{t_{k-1}}}-1\right] \geq(1-a) .
$$

At time $t_{k}$, we have two cases:

$$
\left\{\begin{array}{l}
R_{t_{k}}<1: S_{t_{k}}<S_{t_{k-1}}(S \text { decreases }) \\
R_{t_{k}}>1: S_{t_{k}}>S_{t_{k-1}}(S \text { increases }) .
\end{array}\right.
$$

We assume that the multiple $m_{t_{k-1}}$ is higher than 1 , (thus, it is non negative).

Then, we deduce:

$$
\begin{gathered}
\mathbb{P}^{\mathcal{F}_{t_{k-1}}}\left[m_{t_{k-1}}\left(R_{t_{k}}-1\right)>\frac{L_{t_{k-1}}}{C_{t_{k-1}}}-1\right] \\
=\mathbb{P}^{\mathcal{F}_{t_{k-1}}}\left[m_{t_{k-1}}\left(R_{t_{k}}-1\right)>\frac{L_{t_{k-1}}}{C_{t_{k-1}}}-1 \cap\left(R_{t_{k}}-1\right)>0\right]+ \\
\mathbb{P}^{\mathcal{F}_{t_{k-1}}}\left[m_{t_{k-1}}\left(R_{t_{k}}-1\right)>\frac{L_{t_{k-1}}}{C_{t_{k-1}}}-1 \cap\left(R_{t_{k}}-1\right)<0\right] .
\end{gathered}
$$

- 1.1. If $C_{t_{k-1}}>L_{t_{k-1}}$, then:

$$
\begin{aligned}
& \mathbb{P}^{\mathcal{F}_{t_{k-1}}}\left[m_{t_{k-1}}\left(R_{t_{k}}-1\right)>\frac{L_{t_{k-1}}}{C_{t_{k-1}}}-1\right]= \\
& \mathbb{P}^{\mathcal{F}_{t_{k-1}}}\left[R_{t_{k}}-1>0\right]+\mathbb{P}^{\mathcal{F}_{t_{k-1}}}\left[m_{t_{k-1}}\left(R_{t_{k}}-1\right)>\frac{L_{t_{k-1}}}{C_{t_{k-1}}}-1 \cap\left(R_{t_{k}}-1\right)<0\right]
\end{aligned}
$$

Note that we have:

$$
m_{t_{k-1}}\left(R_{t_{k}}-1\right)>\frac{L_{t_{k-1}}}{C_{t_{k-1}}}-1 \Leftrightarrow R_{t_{k}}>\frac{\frac{L_{t_{k-1}}}{C_{t_{k-1}}}-1}{m_{t_{k-1}}}+1
$$


Then, if the condition $\frac{\frac{L_{t_{k-1}}}{C_{t_{k-1}}}-1}{m_{t_{k-1}}}+1>0$ is fulfilled and we have: $R_{t_{k}}>$ $\frac{\frac{L_{t_{k-1}}}{C_{t_{k-1}}}-1}{m_{t_{k-1}}}+1 .^{5}$

1.1.1. If we have $\frac{\frac{L_{t_{k-1}}}{C_{t_{k-1}}}-1}{m_{t_{k-1}}}+1<0$, then the condition $R_{t_{k}}>\frac{\frac{L_{t_{k-1}}}{C_{t_{k-1}}}-1}{m_{t_{k-1}}}+1$ is satisfied and:

$$
\mathbb{P}^{\mathcal{F}_{t_{k-1}}}\left[1+m_{t_{k-1}} \times \frac{\Delta S_{t_{k}}}{S_{t_{k-1}}}>\frac{L_{t_{k-1}}}{C_{t_{k-1}}}\right]=1>(1-a)
$$

1.1.2. Examine the case $C_{t_{k-1}}>0, C_{t_{k-1}}>L_{t_{k-1}}$ and $\frac{\frac{L_{t_{k-1}}}{C_{t_{k-1}}}-1}{m_{t_{k-1}}}+1>0$.

We have:

$$
\begin{aligned}
& \mathbb{P}^{\mathcal{F}_{t_{k-1}}}\left[m_{t_{k-1}}\left(R_{t_{k}}-1\right)>\frac{L_{t_{k-1}}}{C_{t_{k-1}}}-1\right] \\
= & \mathbb{P}^{\mathcal{F}_{t_{k-1}}}\left[R_{t_{k}}-1>0\right]+\mathbb{P}^{\mathcal{F}_{t_{k-1}}}\left[1>R_{t_{k}}>\frac{\frac{L_{t_{k-1}}}{C_{t_{k-1}}}-1}{m_{t_{k-1}}}+1\right] \\
= & \mathbb{P}^{\mathcal{F}_{t_{k-1}}}\left[R_{t_{k}}>\frac{\frac{L_{t_{k-1}}}{C_{t_{k-1}}}-1}{m_{t_{k-1}}}+1\right],
\end{aligned}
$$

which is equivalent to:

$$
\begin{aligned}
& \mathbb{P}^{\mathcal{F}_{t_{k-1}}}\left[\exp \left[h_{t_{k}}^{-1}\left(\varepsilon_{t_{k}}\right) \sigma_{t_{k}}+\mu_{t}\right]>\frac{\frac{L_{t_{k-1}}}{C_{t_{k-1}}}-1}{m_{t_{k-1}}}+1\right] \geq(1-a) \\
& =\mathbb{P}^{\mathcal{F}_{t_{k-1}}}\left[\varepsilon_{t_{k}}>h_{t_{k}}\left(\frac{1}{\sigma_{t_{k}}} \ln \left(\frac{\frac{L_{t_{k-1}}}{C_{t_{k-1}}}-1}{m_{t_{k-1}}}+1\right)-\frac{\mu_{t_{k}}}{\sigma_{t_{k}}}\right)\right] \geq(1-a) \\
& =1-\mathbb{P}^{\mathcal{F}_{t_{k-1}}}\left[\varepsilon_{t_{k}}<h_{t_{k}}\left(\frac{1}{\sigma_{t_{k}}} \ln \left(\frac{\frac{L_{t_{k-1}}}{C_{t_{k-1}}}-1}{m_{t_{k-1}}}+1\right)-\frac{\mu_{t_{k}}}{\sigma_{t_{k}}}\right)\right] \geq(1-a) \\
& =\mathbb{P}^{\mathcal{F}_{t_{k-1}}}\left[\varepsilon_{t_{k}}<h_{t_{k}}\left(\frac{1}{\sigma_{t_{k}}} \ln \left(\frac{\frac{L_{t_{k-1}}}{C_{t_{k-1}}}-1}{m_{t_{k-1}}}+1\right)-\frac{\mu_{t_{k}}}{\sigma_{t_{k}}}\right)\right] \leq 1-(1-a) .
\end{aligned}
$$

${ }^{5}$ Note that this condition is satisfied as soon as $L_{t_{k-1}}>0$. 
Denote by $N$ the cdf of the standard Gaussian distribution (the cdf of $\varepsilon_{t_{k}}$ ). We have:

$$
N\left[h_{t_{k}}\left(\frac{1}{\sigma_{t_{k}}} \ln \left(\frac{\frac{L_{t_{k-1}}}{C_{t_{k-1}}}-1}{m_{t_{k-1}}}+1\right)-\frac{\mu_{t_{k}}}{\sigma_{t_{k}}}\right)\right] \leq 1-(1-a),
$$

which is equivalent to:

$$
h_{t_{k}}\left(\frac{1}{\sigma_{t_{k}}} \ln \left(\frac{\frac{L_{t_{k-1}}}{C_{t_{k-1}}}-1}{m_{t_{k-1}}}+1\right)-\frac{\mu_{t_{k}}}{\sigma_{t_{k}}}\right) \leq(N)^{-1}(1-(1-a)) .
$$

and also equivalent to:

$$
\frac{\frac{L_{t_{k-1}}}{C_{t_{k-1}}}-1}{m_{t_{k-1}}} \leq \exp \left[\sigma_{t_{k}} h_{t_{k}}^{-1}\left(N^{-1}(1-(1-a))\right)+\mu_{t_{k}}\right]-1 .
$$

Recall that $q_{(\epsilon, T)}=N^{-1}\left[(1-(1-a))^{\frac{1}{T}}\right]$.

Finally, we have:

1-1) If $\exp \left[h_{t_{k}}^{-1}\left(q_{(a, T)}^{\varepsilon}\right) \sigma_{t_{k}}+\mu_{t_{k}}\right]<1$

If $\frac{L_{t_{k-1}}}{C_{t_{k-1}}}<\exp \left[h_{t_{k}}^{-1}\left(q_{(a, T)}^{\varepsilon}\right) \sigma_{t_{k}}+\mu_{t_{k}}\right]$, then $m_{t_{k-1}}$ must satisfy the following constraint:

$$
m_{t_{k-1}} \leq \frac{\frac{L_{t_{k-1}}}{C_{t_{k-1}}}-1}{\exp \left[h_{t_{k}}^{-1}\left(q_{(a, T)}^{\varepsilon}\right) \sigma_{t_{k}}+\mu_{t_{k}}\right]-1} .
$$

If $\frac{L}{C_{t_{k-1}}}>\exp \left[h_{t_{k}}^{-1}\left(q_{(a, T)}^{\varepsilon}\right) \sigma_{t_{k}}+\mu_{t_{k}}\right]$, then the previous constraint must be satisfied but also involves more that $m_{t_{k-1}}<1$.

1-2) If $\exp \left[h_{t_{k}}^{-1}\left(q_{(a, T)}^{\varepsilon}\right) \sigma_{t_{k}}+\mu_{t_{k}}\right]>1$, then ther is no constraint for $m_{t_{k-1}}$. Case 2: $0<C_{t_{k-1}}<L_{t_{k-1}}$, Therefore: $L_{t_{k-1}}>0$.

As previously, the quantile condition is given by:

$$
\mathbb{P}^{\mathcal{F}_{t_{k-1}}}\left[1+m_{t_{k-1}} \times \frac{\Delta S_{t_{k}}}{S_{t_{k-1}}}>\frac{L_{t_{k-1}}}{C_{t_{k-1}}}\right] \geq(1-a),
$$

which is equivalent to:

$$
\mathbb{P}^{\mathcal{F}_{t_{k-1}}}\left[m_{t_{k-1}}\left(R_{t_{k}}-1\right)>\frac{L_{t_{k-1}}}{C_{t_{k-1}}}-1\right] \geq(1-a)
$$

At time $t_{k}$, we have two cases:

$$
\left\{\begin{array}{l}
R_{t_{k}}<1: S_{t_{k}}<S_{t_{k-1}}(S \text { decreases }) \\
R_{t_{k}}>1: S_{t_{k}}>S_{t_{k-1}}(S \text { increases }) .
\end{array}\right.
$$


We assume that the multiple $m_{t_{k-1}}$ is non negative.

$$
\begin{aligned}
& \mathbb{P}^{\mathcal{F}_{t_{k-1}}}\left[m_{t_{k-1}}\left(R_{t_{k}}-1\right)>\frac{L_{t_{k-1}}}{C_{t_{k-1}}}-1\right] \\
= & \mathbb{P}^{\mathcal{F}_{t_{k-1}}}\left[m_{t_{k-1}}\left(R_{t_{k}}-1\right)>\frac{L_{t_{k-1}}}{C_{t_{k-1}}}-1 \cap\left(R_{t_{k}}-1\right)>0\right]+ \\
& \mathbb{P}^{\mathcal{F}_{t_{k-1}}}\left[m_{t_{k-1}}\left(R_{t_{k}}-1\right)>\frac{L_{t_{k-1}}}{C_{t_{k-1}}}-1 \cap\left(R_{t_{k}}-1\right)<0\right] .
\end{aligned}
$$

Therefore, we have:

$$
\begin{aligned}
& \mathbb{P}^{\mathcal{F}_{t_{k-1}}}\left[m_{t_{k-1}}\left(R_{t_{k}}-1\right)>\frac{L_{t_{k-1}}}{C_{t_{k-1}}}-1\right] \\
= & \mathbb{P}^{\mathcal{F}_{t_{k-1}}}\left[m_{t_{k-1}}\left(R_{t_{k}}-1\right)>\frac{L_{t_{k-1}}}{C_{t_{k-1}}}-1 \cap\left(R_{t_{k}}-1\right)>0\right] \\
= & \mathbb{P}^{\mathcal{F}_{t_{k-1}}}\left[m_{t_{k-1}}\left(R_{t_{k}}-1\right)>\frac{L_{t_{k-1}}}{C_{t_{k-1}}}-1\right] .
\end{aligned}
$$

Consequently, we get the equivalence between the two following conditions:

$$
\begin{gathered}
\mathbb{P}^{\mathcal{F}_{t_{k-1}}}\left[1+m_{t_{k-1}} \times \frac{\Delta S_{t_{k}}}{S_{t_{k-1}}}>\frac{L_{t_{k-1}}}{C_{t_{k-1}}}\right] \geq(1-a), \\
\mathbb{P}^{\mathcal{F}_{t_{k-1}}}\left[R_{t_{k}} \geq \frac{\frac{L_{t_{k-1}}}{C_{t_{k-1}}}-1}{m_{t_{k-1}}}+1\right] \geq(1-a) .
\end{gathered}
$$

This is equivalent to:

$$
\mathbb{P}^{\mathcal{F}_{t_{k-1}}}\left[R_{t_{k}} \geq \frac{\frac{L_{t_{k-1}}}{C_{t_{k-1}}}-1}{m_{t_{k-1}}}+1\right] \geq(1-a)
$$

Thus, the quantile condition is equivalent to:

$$
\mathbb{P}^{\mathcal{F}_{t_{k-1}}}\left[\varepsilon_{t_{k}}>h_{t_{k}}\left(\frac{1}{\sigma_{t_{k}}} \ln \left(\frac{\frac{L_{t_{k-1}}}{C_{t_{k-1}}}-1}{m_{t_{k-1}}}+1\right)-\frac{\mu_{t_{k}}}{\sigma_{t_{k}}}\right)\right] \geq(1-a)
$$

And also to:

$$
h_{t_{k}}\left(\frac{1}{\sigma_{t_{k}}} \ln \left(\frac{\frac{L_{t_{k-1}}}{C_{t_{k-1}}}-1}{m_{t_{k-1}}}+1\right)-\frac{\mu_{t_{k}}}{\sigma_{t_{k}}}\right) \leq N^{-1}(1-(1-a)) .
$$

We obtain:

$$
\frac{\frac{L_{t_{k-1}}}{C_{t_{k-1}}}-1}{m_{t_{k-1}}} \leq \exp \left[h_{t_{k}}^{-1}\left(q_{(a, T)}^{\varepsilon}\right) \sigma_{t_{k}}+\mu_{t_{k}}\right]-1 .
$$


Finally, we have:

2-1) If $\exp \left[h_{t_{k}}^{-1}\left(q_{(a, T)}^{\varepsilon}\right) \sigma_{t_{k}}+\mu_{t_{k}}\right]<1$, there is no positive solution for $m_{t_{k-1}}$

2-2) If $\exp \left[h_{t_{k}}^{-1}\left(q_{(a, T)}^{\varepsilon}\right) \sigma_{t_{k}}+\mu_{t_{k}}\right]>1$, then :

If $\frac{L_{t_{k-1}}}{C_{t_{k-1}}}<\exp \left[h_{t_{k}}^{-1}\left(q_{(a, T)}^{\varepsilon}\right) \sigma_{t_{k}}+\mu_{t_{k}}\right]$, then there is no constraint for $m_{t_{k-1}} \quad$ If $\frac{L_{t_{k-1}}}{C_{t_{k-1}}}>\exp \left[h_{t_{k}}^{-1}\left(q_{(a, T)}^{\varepsilon}\right) \sigma_{t_{k}}+\mu_{t_{k}}\right]$, then $m_{t_{k-1}}$ must satisfy the following constraint:

$$
m_{t_{k-1}} \geq \frac{\frac{L_{t_{k-1}}}{C_{t_{k-1}}}-1}{\exp \left[h_{t_{k}}^{-1}\left(q_{(a, T)}^{\varepsilon}\right) \sigma_{t_{k}}+\mu_{t_{k}}\right]-1} .
$$

